\title{
Cross-Reactive Anti-Avian H5N1 Influenza Neutralizing Antibodies in a Normal 'Exposure-Naïve' Australian Blood Donor Population
}

\author{
Garry W. Lynch ${ }^{*}, 1,2$, Paul W. Selleck ${ }^{3}$, Anna-Maree Axell ${ }^{3}$, Teena Downton ${ }^{1}$, Natalie M. Kapitza ${ }^{1}$, \\ Ingrid Boehm ${ }^{2}$, Wayne Dyer ${ }^{1,2}$, Ying-Fan Yvonne Wang ${ }^{1}$, Sacha Stelzer-Braid ${ }^{4}$, \\ William Rawlinson ${ }^{4}$ and John S. Sullivan ${ }^{1,2}$
}

\author{
${ }^{I}$ The Transfusion Immunobiology and Biosafety Group, Transfusion Medicine and Immunogenetics Research, The \\ University of Sydney, Camperdown, Sydney, Australia \\ ${ }^{2}$ Research \& Business Development, The Australian Red Cross Blood Service, 153 Clarence St, Sydney, NSW 2000, \\ Australia \\ ${ }^{3}$ The Australian Animal Health Laboratories, CSIRO, Geelong, Victoria, Australia \\ ${ }^{4}$ Virology Division, Department of Microbiology, South Eastern Area Laboratory Service, Prince of Wales Hospital, \\ Randwick, NSW 2031, Australia
}

\begin{abstract}
It is necessary to understand whether some humans possess natural humoral-immune protection for avianH5N1 influenza. To broadly assess an exposure naïve cohort we have examined intravenous immunoglobulins (IVIGs) isolated from pools of many thousands of normal Australian blood donations. In studies of the anti-H5N1 antibody potential of these highly purified IVIG therapeutics and of individual donor sera we have identified antibodies that bind to both H5N1 surface envelope and internal viral proteins and neutralize in vitro MDCK and Vero cell infections by highly pathogenic avian influenza clade I and II and human-derived H5N1 isolates. As this reactivity is removed by adsorption with purified $\mathrm{H} 3 \mathrm{~N} 2$ and $\mathrm{H} 1 \mathrm{~N} 1$ strains, anti-H5N1 cross-reacting hetero-typic antibodies are implicated. These findings support that some individuals do contain low levels of specific and neutralizing anti-H5N1 antibodies. The protective relevance of this in vivo remains yet to be determined.
\end{abstract}

Keywords: Avian H5N1 influenza, human anti-viral antibodies, infection neutralization, virus, IVIG.

\section{INTRODUCTION}

Fed by high mutation rates and gene re-assortments Influenza strains can escape host homotypic antibody defenses. Yearly, genetic drift in human influenza strains generates epidemics that mainly target the immune-compromised, particularly the very young and the elderly, and result in the deaths of approximately $0.1 \%$ of those infected with up to 250-500 thousand lives lost every year [1]. Three to four times a century, genetic shifts promote the emergence of avian influenza strains of altered phenotypes, cell tropisms and pathogenicities that enable them to transverse the species barrier and seed highly lethal human pandemics. Notable is that pandemic influenza particularly targets otherwise healthy youths as well as the immune-compromised. During the $1918 \mathrm{H} 1 \mathrm{~N} 1$ pandemic, local mortalities of $30 \%$ and more were recorded, and an overall mortality of approximately $2.6 \%$ with up to 50 million lives lost $[2,3]$. The epizoonotic expansion over the past decade of highly pathogenic H5N1 avian-influenza (HPAI) has so far resulted in a $61.8 \%$ mortality of the confirmed 348 human infections (WHO) identified up till the beginning of 2008 and continues to pose a realistic risk of also developing into a human pandemic [4]. As a significant proportion of infected individuals confirmed

*Address correspondence to this author at The Transfusion Immunobiology and Biosafety Group, Level 8 R\&BD Laboratories, The Australian Red Cross Blood Service, 153 Clarence St Sydney, NSW 2000, Australia; Tel: 61-2-9519 8151; E-mail: garryVilynch@optusnet.com.au by laboratory tests have survived exposure to $\mathrm{H} 5 \mathrm{~N} 1$, as did the majority of the populations through the earlier 1918, 1957 and 1968 pandemics, we have asked whether there is some cross-reactive immune protection in the population at large. Since neutralizing antibodies remain the current best single protective correlate for influenza [5], we aimed to determine if there exists cross-reactive anti-H5N1 antibodies gained from broad lifetime exposures to various human influenza A strains that may provide a level of protection. Our findings challenge the conventional view that an exposure naïve population is void of neutralizing antibodies against avian H5N1 influenza.

\section{MATERIALS AND LABORATORY PROCEDURES}

\section{Antibodies}

Intravenous Immunoglobulins (IVIGs) derived from plasma collected by the Australian Red Cross Blood Service from thousands of non-re-numerated Australian blood donors were examined to broadly assess the potential repertoire of anti-H5N1 antibodies (Abs) in the Australian population. Each IVIG batch was prepared by large pool fractionation and purification by CSL Pty Ltd into the highly pure $(\geq 98 \%)$ Intragam-P ${ }^{\mathrm{TM}}$ IVIG formulation. This antibody was sourced from the plasmas of up to 60,000 normal blood donors and provides a broad antibody sampling of the herd immunity of the donor population. Dilution of the IVIG for our experiments with TBS or PBS is indicated in the text. We have also examined the antibodies of individual and pooled serum obtained from children (aged 1-4 years) or adults of varying 
age group (e.g., 11-20, 21-30, 31-40, 41-50, 51-60, 61-70, 71-80 years).

\section{Viruses}

The avian influenza strains examined were three $\mathrm{H} 5 \mathrm{~N} 1$ strains, namely; clade1 strains A/ chicken/Vietnam/8/2004 (GenBank accession number AY724788) and A/human/Vietnam/1203/2004 (GenBank \# EF541403), and a clade2 strain $\mathrm{A} /$ chicken/Wates/1/2005 (GenBank \#EU124055); and a H7N7 strain, namely; A/chicken/Victoria/1985 (GenBank \#CY025069). The avian viruses were grown in the allantoic sac of embryonated chicken eggs and purified on sucrose gradients. Briefly, allantoic fluid was collected from infected eggs, pooled and clarified by low speed centrifugation. Virus was pelleted in an ultracentrifuge, resuspended and purified by ultracentrifugation through a $20-50 \%$ discontinuous sucrose density gradient. The visible band was collected, the virus pelleted in an ultracentrifuge and resuspended in tris buffered saline (TBS).

The growth and expansion of the HPAI avian viruses was performed at the CSIRO Australian Animal Health Laboratories (AAHL), Geelong, Victoria. To permit removal from the AAHL secure area and to allow the virus to be handled safely for antibody binding and protein analyses the viruses were inactivated by exposure to 5 megarads of radiation. The purity of the viruses was qualified by their SDS-PAGE profiles (see below).

The representative human viruses examined in this study were chicken egg expanded human vaccine strain isolates, namely; two human $\mathrm{H} 1 \mathrm{~N} 1$ isolates; A/Texas/36/1991 and A/Johannesburg/82/1996; and two human H3N2 viruses, $A /$ Beijing/32/1992 and A/Sydney/5/1997. These were kindly provided by Dr A Hampson, WHO laboratory, Parkville, Victoria, Australia. These isolates are antigenically representative of viruses that have circulated in recent times and have been included in northern and southern hemisphere influenza vaccines between 1992 and 2000 .

\section{Infection Studies}

As HPAI H5N1 influenza is a Biosecurity level 3 organism all experiments utilizing live virus were performed in the high security laboratories at AAHL. Virus neutralization assays were done in MDCK (Madin-Darby Canine Kidney - ATCC CCL-34) cells or Vero (African green monkey kidney ATCC CCL-81) using a modification of a standard protocol [6]. Briefly, 100 tissue culture infectious doses of each virus was added to two-fold dilutions of each serum in microtitre plate wells and incubated for 60 minutes at $37^{\circ} \mathrm{C}$. Cells were added and the plates incubated at $37^{\circ} \mathrm{C}$ for 3 days. The addition of trypsin was not necessary as all viruses used in this trial had a multi-basic cleavage site in the hemagglutinin. The neutralizing titre of a serum was the highest dilution of serum showing complete neutralization of the virus.

\section{Electrophoresis, Western (WB) and Spot (SB) Blotting}

Viruses were subjected to SDS detergent solubilization and electrophoresis on criterion (Biorad, Life Sciences, Hercules, Ca) 4-16\% gradient gels (SDS-PAGE) under nonreducing conditions as described earlier [7]. Where indicated SDS-PAGE separated viral proteins were identified by Coomassie blue gel staining
Alternately the electrophoresed viral proteins were electrotransfered onto $0.2 \mathrm{um}^{\text {Protran }}{ }^{\text {reg }}$ nitrocellulose (Schleicher and Schuell, Whatman, PE- Life \& Analytical Sciences, Boston, MA), followed by blocking with a $5 \%$ dried milk-powder in TBS solution. Western Blotting (WB) of these was performed using 1/200-1/500 dilutions of IVIG $(50 \mathrm{mg} / \mathrm{ml})$, with incubation for $1 \mathrm{hr}$ at room temperature (RT). After washing with TBS, blots were incubated at RT for $1 \mathrm{hr}$ with a 1/40,000 dilution of a horseradish peroxidase (HRP) goat anti-whole human antibody (Sigma-Aldrich Chemicals, MO). Followed by washing to remove unbound secondary $\mathrm{Ab}$, and bound antibody identified by chemiluminescence (HRP detection kit, Amersham Biosciences UK Ltd. Buckinghamshire, England) [7].

For studies of native whole virus $1 \mu 1-2 \mu 1$ spots of tenfold serial dilutions of $500 \eta \mathrm{g}$ of the respective isolates were arrayed on nitrocellulose. Similarly protein controls of tenfold serial dilutions of $500 \eta \mathrm{g}$ of human albumin were also arrayed.

\section{Antibody Depletion Studies}

For this $2 \mu \mathrm{l}$ spotted adsorption panels of $12 \times 1 \mu \mathrm{g}$ of each of the A/Beijing/32/1992 and A/human/Sydney/5/92 $\mathrm{H} 3 \mathrm{~N} 2$ isolates were immobilized to nitrocellulose. Proteinfree sites were blocked using 5\% skimmed-milk protein diluted in TBS (as for the WBs above). For comparative examination of the binding Abs of children's and adult serum pools, these were first pre-exposed (i.e. adsorbed serum) or non-exposed to the adsorption panel. Bound Abs from each of the serum sets with or without pre-exposure to H3N2 virus were then tested against serial dilutions of the whole human avian virus panel arrayed as for the SB studies above. Virus protein loadings were equalized for tenfold dilutions of virus starting at $500 \eta \mathrm{g}$ with up to $10^{7}$ fold dilutions as indicated. Antibody binding to the respective panels was detected following secondary HRP-Ab conjugate treatment and chemiluminescence as for the above WB studies.

\section{RESULTS}

We determined whether IVIGs could neutralize the infection of MDCK or Vero cells by H5N1 viruses in order to test our hypothesis that humans have anti-H5N1 specific antibodies.

\section{H5N1 Infection Neutralization}

In eight separate micro-neutralization experiments we have consistently observed blanket inhibition of MDCK or Vero cells infections by HPAI clade I and II chicken-derived viruses and a clade I human-derived strain, when the viruses where first pre-treatment with Australian-derived IVIG (Table 1). This data represents a broader panel of experimental findings with the additional support of parallel neutralization of each of the H5N1 isolates using 3 other IVIG-Ab formulations, sourced from Europe, the US and SE-Asia (data not shown). This also clearly and consistently demonstrates neutralization of $\mathrm{H} 5 \mathrm{~N} 1$ cell culture infections by IVIG-Abs. It was noteworthy that in marked contrast to the H5N1 neutralization by the IVIGs they all have failed to inhibit Vero cell infection by a H7N7 HPAI virus (i.e. A/chicken/Victoria/1985) (Table 1). This indicates a direct specific neutralization of H5N1 by the highly purified antibodies in IVIG instead of an indirect action of IVIG effects on cell mediated 
Table 1. Australian IVIG Antibody Neutralization of Avian H5N1 Cell Culture Infections

\begin{tabular}{|l|c|c|}
\hline & Vero Cells & MDCK Cells \\
\hline Virus & Neutralization Titre & Neutralization Titre \\
\hline \hline Avian H5N1 Influenzas & $8-32^{1}$ & $8-16^{2}$ \\
\hline Chicken/Vietnam/8/2004*(Clade 1$)$ & 32 & 1024 \\
\hline Chicken/Wates/1/2005 (Clade II) & $32^{1}$ & 128 \\
\hline Human/Vietnam/1203/2004 (Clade I) & & \\
\hline Avian H7N7 Influenza & $<2^{1}$ & ND \\
\hline Chicken/Victoria/1985 & \multicolumn{2}{|c|}{} \\
\hline
\end{tabular}

The data from 8 separate neutralization experiments have been collated and the antibody inhibition titers are listed. For this Australian prepared IVIG was assayed in duplicates against chicken-derived H5N1 clade I and II strains, a human-derived strain and a duck H7N7 virus. The values obtained are given as the reciprocal of the highest dilution that gave complete neutralization against $100 \mathrm{TCID}_{50}$ of each of the low passage HPAI Influenza A isolates. Note: This was also supported in each experiment by parallel neutralization of each of the isolates by three other IVIG-Ab formulations, sourced from Europe, the US and SE-Asia. Those IVIGs also failed to inhibit H7N7 infections (data not shown).

${ }^{1}$ Titre value/range in 2 separate infection experiments.

${ }^{2}$ Titre range from 6 separate infections.

responses. Partial neutralization with breakthrough virus was found to extend for several dilutions beyond the listed titres that gave complete neutralization.

\section{Human Antibodies Bind to Denatured Surface Envelope and Internal H5N1 Proteins}

To biochemically identify the viral proteins targeted by IVIG-Abs, the purified viruses were solubilized by Sodium Dodecyl Sulfate (SDS) and the extracted viral proteins subjected to electrophoresis (PAGE). Followed by staining to profile the proteins of the human and avian influenza viruses (Fig. 1, middle panel lanes 2-6). Identification of only viral proteins [8] indicates the purity of the respective viruses isolated free of egg culture proteins. As predicted each of the viral proteins from the respective isolates migrate with equivalent mobility's in SDS-PAGE. The hemagglutinins (HA) were the standout exception with relative gel mobility ranking differences of HA5 $>$ HA1 $>$ HA3. Observed apparent molecular weights of 72,77 and $82 \mathrm{kDa}$ for the respective hemagglutinin monomers of HA5, HA1 and HA3 were found and indicative of differential HA glycosylation between those strains [7]. SDS-PAGE fractionated viral proteins separated in the same gel were alternately electrotransferred to nitrocellulose and WB performed using Australian-sourced IVIG [7]. When matched against the viral protein signatures (Fig. 1, lanes 2-6) the WB profiles (lanes 8 -9) revealed clear human-Ab binding to denatured (linear) epitopes in human influenza envelope HA and NA and internal NP2, M1 and NS2 viral proteins (Fig. 1, lanes 8 \& 9). Most relevant was parallel antibody binding to the corresponding NP2, M1 and NS2 proteins of H5N1 (lane 7). Technical refinement, including altering the detergent in the blotting reaction, previously found to effect WB detection of viral proteins[9], subsequently enabled resolution of IVIG$\mathrm{Ab}$ binding to $\mathrm{H} 5 \mathrm{~N} 1 \mathrm{HA}, \mathrm{NA}$ and M2 proteins (lane 1). However, a caveat needs to be raised regarding the long held perception that antibodies bind in western blots to only linear epitopes, in the light of recent findings by Zhou et al. that challenge this view, with evidence that Ab-binding to discontinuous regions can occur. They have suggested that this is possible as a result of protein refolding of secondary structures during gel-blot processing [21].

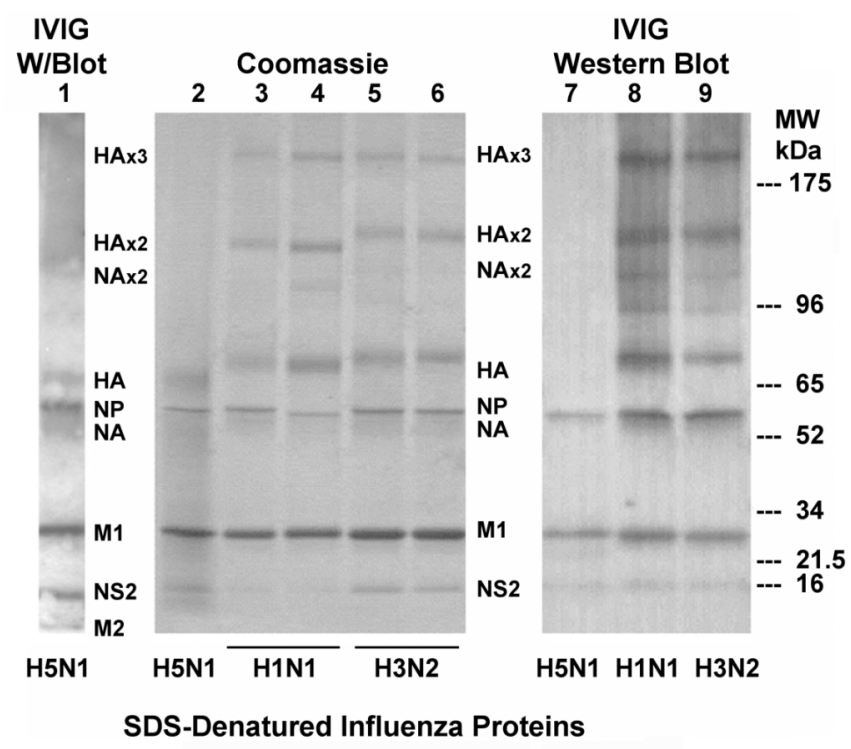

Fig. (1). Detection of Antibody binding to influenza antigen in Australian Intravenous Immunoglobulin (IVIG). Coomassie Stained Center Panel. Viruses were subjected to SDS-PAGE under non-reducing conditions. Avian H5N1 lane 2: A/chicken/Vietnam/8/'04; Human H1N1 viruses: lane 3: A/Texas/36/1991 and lane 4: A/Johannesburg/82/1996; Human H3N2 viruses: lane 5: A/Beijing/32/1992 and lane 6: A/Sydney/5/1997 viral strains. The purity of each of the virus preparations was evident from coomassie blue gel staining which clearly revealed the well established profiles of most abundant proteins in the respective isolates; namely Hemagglutinin (HA) (and multimers) (500 copies per virion), Neuraminidase (NA) (100 copies), Nucleoprotein (NP) (1,000 copies), M1 (3,000 copies) and NS2 protein (130-200 copies) [8]. IVIG Western Blot Panels. Alternately WB with a $1 / 250$ dilution of IVIG $(50 \mathrm{mg} / \mathrm{ml}, 1 \mathrm{hr}$ incubation at RT), revealed human antibody binding to the viral proteins HA, NA, NP, M1 and NS2 of both human (H1N1 (lane 8) and H3N2 (lane 9)) and avian (H5N1) influenza (lane 1 was blotted in TBS without tween-20; and lane 7 blotted in TBS with $0.1 \%$ tween-20). For direct comparison of the blotting and protein staining results these were performed in the same SDSPAGE gel experiment (i.e. Fig. (1B) middle and right hand panels (lanes 2-9)). 


\section{Human Antibodies Bind to the Envelope Surface of In- tact H5N1 Virus}

To further evaluate the anti-H5N1 antibodies in neutralization competent IVIG we performed IVIG-WB assays on whole avian-H5N1 and human-H1N1 and H3N2 strains immobilized to nitrocellulose for evidence of antibody binding to native H5N1 virus (Fig. 2). Serial dilutions of purified human influenzas are shown with IVIG antibody binding to intact virus titred out to a 1/1000 dilution (Fig. 2, top panel). IVIG-Ab binding to intact $\mathrm{H} 5 \mathrm{~N} 1$ was also evident for viruses immobilized to nitrocellulose (Fig. 2, lower panel-with nitrocellulose loading equalized with the human viruses), but at titres 100-1000 fold less than for the equivalent load of H1N1 or H3N2 viruses. Collectively our findings of anti$\mathrm{H} 5 \mathrm{~N} 1$ binding and neutralizing antibodies in human 'herd' IVIG preparations suggest that some individuals within the normal population have antibody activities (neutralization) directed to envelope $\mathrm{H} 5 \mathrm{~N} 1$ and to several denatured surface and internal viral proteins.

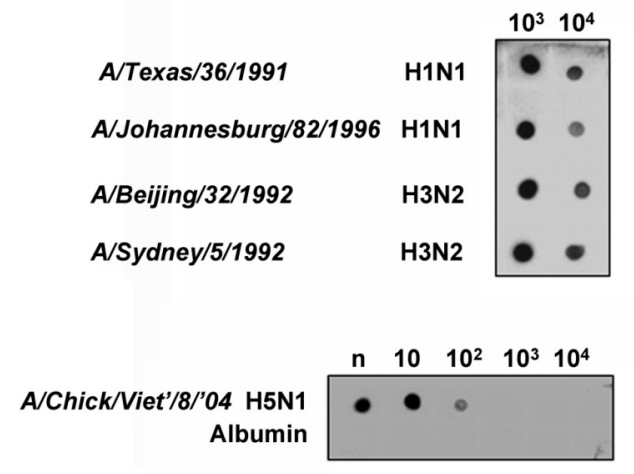

IVIG Dot Blots of Intact Influenzas

Fig. (2). Detection of human antibodies in Australian IVIG that bind native influenza antigen of whole virions. For examination of IVIG-Antibody binding to the envelope proteins of the intact viruses $2 \mu$ samples of whole virus and titrations thereof were spotted onto nitrocellulose. H5N1, H3N2 and H1N1 viral loadings were equalized for total protein and matched against control samples of human albumin. Ab binding was revealed by secondary HRP-Ab treatment and chemiluminescence [7].

\section{Human Ab Binding to H5N1 is Proportional to Influenza Exposure}

If human $\mathrm{Ab}$ binding to $\mathrm{H} 5 \mathrm{~N} 1$ is the result of nonspecific interactions, we would predict that serum Abs from a population minimally exposed to influenza, such as children, would bind to $\mathrm{H} 5 \mathrm{~N} 1$ virus equivalent to the serum Abs of adults. Therefore we compared the Abs in an adult serum pool (10 sera: ages 31 to 40 years) with pooled children's serum (10 sera of ages 1 to 4 years) for binding to whole $\mathrm{H} 5 \mathrm{~N} 1$ or Human $\mathrm{H} 1 \mathrm{~N} 1$ or $\mathrm{H} 3 \mathrm{~N} 2$ viruses immobilized to nitrocellulose (Fig. 3). As expected, there was marked adult serum $\mathrm{Ab}$ binding to $\mathrm{H} 1 \mathrm{~N} 1$ and $\mathrm{H} 3 \mathrm{~N} 2$ proteins gained from 30-40 years of repeated exposures to various different influenza A strains. But considerably less Ab binding (10 to 100 fold less) detected for the children's sera, indicative of limited human influenza A exposures for the children. We then observed minimal evidence of $\mathrm{Ab}$ binding for $\mathrm{H} 5 \mathrm{~N} 1$ in the children's sera, but clear binding of adult serum Abs to
$\mathrm{H} 5 \mathrm{~N} 1$ which was markedly less than to the $\mathrm{H} 1 \mathrm{~N} 1$ or $\mathrm{H} 3 \mathrm{~N} 2$ strains. This indicated that the observed human Ab binding to $\mathrm{H} 5 \mathrm{~N} 1$ was age and influenza exposure-dependent, rather than the result of unrelated non-specific binding. In parallel studies, we have also found evidence for specific adult serum Abs that bind to gel-separated denatured linear H5N1 epitopes, similar to our findings for IVIG, again with minimal evidence for antibody binding from two pools of children's sera (Fig. 4).
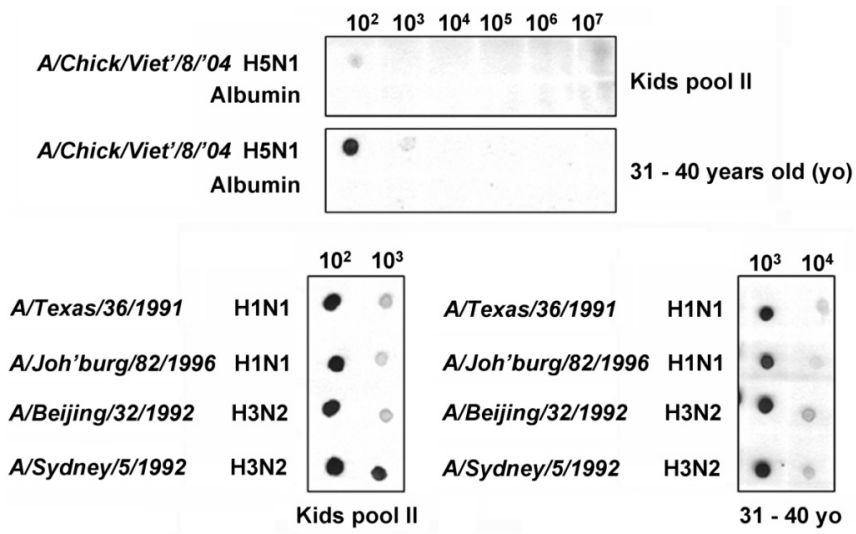

Adult vs Child Serum Dot Blots of Intact Influenzas

Fig. (3). Comparisons of anti-H5N1 antibodies in child (age 1 to 4 years) or adult (age 31 to 40 years) pooled sera. For identification of anti-influenza antibodies in adult and child sera dot blotting was performed as for Fig. (2) applying whole purified $\mathrm{H} 5 \mathrm{~N} 1$ (A/chicken/Vietnam/8/2004), H1N1 (A/human/Texas/36/1991; A/ human/Johannesberg/82/96) and H3N2 (A/human/Beijing/32/1992 and $\mathrm{A} / \mathrm{human} / \mathrm{Sydney} / 5 / 92$ ) viruses and using 1/500 dilutions of a pools of child $(\mathrm{x} 10)$ or adult $(\mathrm{x} 10)$ sera. The top panel examines binding to $\mathrm{H} 5 \mathrm{~N} 1$ and the bottom panels, comparative antibody binding to equalized titrations of the human H1N1 and H3N2 influenza isolates. Note: The dot blots were all performed in parallel within the same blotting experiment, and reveal adult serum antibodies that bind to the envelope proteins of intact H5N1 virus.

\section{Proportional Hetero-Subtypic Depletion of Anti-Human Influenza and Anti-Avian H5N1 Abs Following Prior- Exposure to Whole Human H3N2 Virus}

We have performed viral-Ab clearance studies to determine the inter-virus specificity of the observed anti-H5N1 activity. As shown in Fig. (5) pre-exposure of an adult serum pool to human H3N2 influenza selectively and proportionally removes both anti-human influenza and anti-avian binding Abs. This indicates firstly that these Abs are specific for influenza, and secondly that the anti-H5N1 human Abs have arisen not from the unlikely event of sub-clinical exposure to avian $\mathrm{H} 5 \mathrm{~N} 1$ virus but from repeated exposure to heterogeneous influenza challenge per se. From these findings and from other data not shown, we have found that depletion of human Abs in IVIGs or serum can be performed irrespective of whether we use the same H5N1 strain; such as a Viet/8 (i.e. same subtype); or a subtype different $\mathrm{H} 5 \mathrm{~N} 1$ virus, such as with pre- adsorption of Abs-to Viet/8 using a Viet/1203 virus (i.e. different subtype); or as we have as indicated in Fig. (5) pre-adsorbed with an unrelated human-H3N2 influenza strain (i.e. different strain) (Fig. 5). When antibody quantitation was assessed by spot titration of the total immunoglobulin in the samples pre- and post adsorption there was no 
quantitative loss of serum antibodies concurrent with depletion of the cross-reactive anti-H5N1 antibodies, thus showing the specificity of the latter.

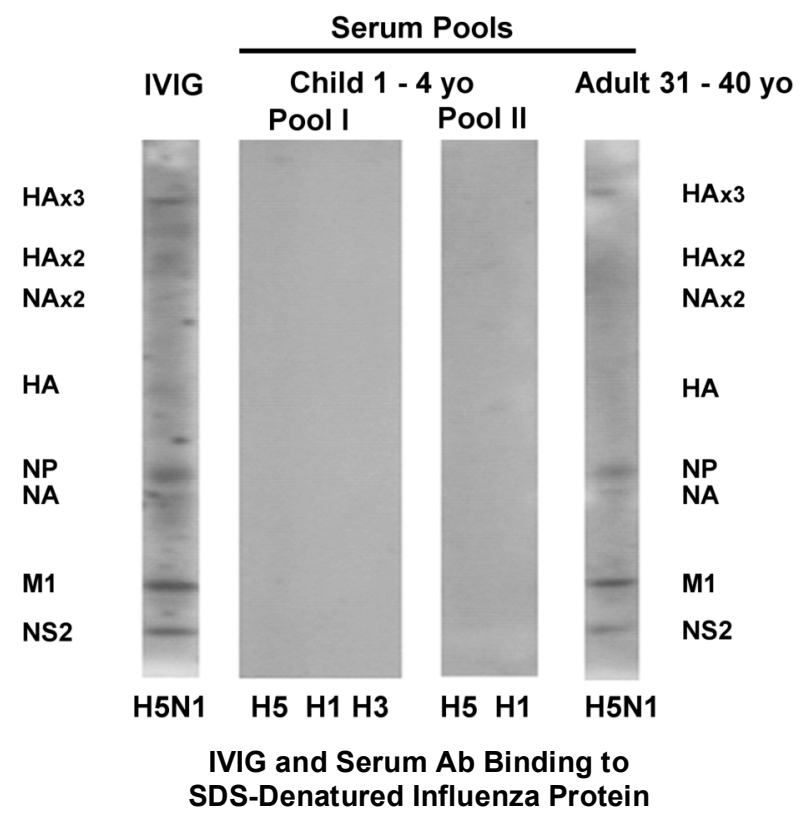

Fig. (4). Comparisons of anti-H5N1 antibodies in IVIGs and pooled sera of children (1-4 years) and adults (31-40 years) for the binding to denatured human and avian H5N1 influenza proteins. Shown are the western blot profiles obtained using different aged sera and Australian-derived IVIG for binding to denatured human $\mathrm{H} 3 \mathrm{~N} 2$ and $\mathrm{H} 1 \mathrm{~N} 1$ and avian $\mathrm{H} 5 \mathrm{~N} 1$ proteins. Western blotting was performed as for Fig. (2) for purified H5N1 (A/chicken/Vietnam/8/2004); H1N1 (A/human/Johannesberg/82/96) and H3N2 (A/human/Sydney/5/92) viruses using a $1 / 250$ dilution of IVIG (50 $\mathrm{mg} / \mathrm{ml}$ ), or a $1 / 500$ dilution of a pool (i.e. I x3 and II x10) of children or adult (x10) sera. When processed in parallel it can be seen that under conditions that reveal antibody binding to H5N1 HA, NA/NP, M1 and NS2 proteins there is minimal antibody binding for either of the children serum pools.

\section{Summary of our Findings that Show Ab Specificity for H5N1 from Unexposed Humans}

Our findings that show anti-H5N1 human Abs in exposure naïve Australian blood have been qualified by a number of different and complementary means, namely by; 1) cell culture neutralization, 2) solid-phase immuno-assay of $\mathrm{Ab}$ binding to whole virus and 3) western blotting assays, all of which were confirmatory and clearly identify low level human Abs that bind to $\mathrm{H} 5 \mathrm{~N} 1$ and protect against cell infection by multiple avian $\mathrm{H} 5 \mathrm{~N} 1$ isolates in cell culture. In these studies establishment of the specificity of H5N1 interactions were satisfied by demonstration of; i) titratable Ab-binding interactions; ii) complementary binding of either normal adult human serum Abs or of highly purified IVIG-Abs; iii) $\mathrm{Ab}$ binding to highly purified preparations of $\mathrm{H} 5 \mathrm{~N} 1$, iv) and binding to both denatured and native $\mathrm{H} 5 \mathrm{~N} 1$ protein preparations; v) with minimal binding of children's serum Abs to denatured or intact $\mathrm{H} 5 \mathrm{~N} 1$ virus (Figs. 1-4); vi) a lack of neutralization of an avian H7N7 isolate studied in parallel with H5N1 neutralization, and vii) the specific depletion of binding activities of IVIGs or normal serum Abs with whole virus or denatured viral proteins by $\mathrm{Ab}$ pre-clearing treatments
(Fig. 5). In addition, in serological tests using a commercial ELISA anti-H5 mAb Hemagglutinin competition assay we have also detected viii) anti-H5 HA binding in the serum of some individuals and this activity was removed on adsorption with H5N1 viruses (S. Stelzer, B. Wong, W. Rawlinson, G. Lynch unpublished observations).

\section{DISCUSSION}

We provide direct evidence for bona fide specific and protective cross-reactive anti-H5N1 antibodies in a previously unexposed population. This activity clearly differs from the more marked Ab-activities observed in $\mathrm{H} 5 \mathrm{~N} 1$ vaccine responses [1] and from convalescent $\mathrm{H} 5 \mathrm{~N} 1$ infected individuals [10] and the modest in vitro activities we detect may often be masked within the low and perhaps equivocal value ranges of some assay. We have validated our observations using multiple different assays and our findings gain support from recent reports of anti-NA antibodies in normal unexposed serum [11] and of enhanced anti-H5N1 HA neutralization in healthy donors after boosting with unrelated human influenza H1N1/ H3N2 seasonal vaccines [12]. Less direct evidence has also been gained from indication of H5N1 immune protection in aged individuals [13], and from animal studies, as summarized in the bioinformatics evaluations of Bui et al. [5], that show the conservation of numerous linear and conformational epitopes (some neutralizing) across H1N1, H3N2 and H5N1 strains. The low levels of human antibodies we find bind to $\mathrm{H} 5 \mathrm{~N} 1$ viruses, orders of magnitude less than for human strains. It is important therefore to determine whether these cross-reactive antibodies could contribute to the observed age-related survival of the $40 \%$ individuals that have been infected with H5N1 [4], perhaps through mechanisms that subdue viral infection and expansion thereby protecting those individuals against clinical disease.

Our findings and those identified in [5] suggest that functional epitope-specific Ab-targets can be maintained and conserved across viral species. Indicated is a need to focus on viral epitope structural interactions. To assist in understanding influenza immune capture and escape there are new methods for analyzing protein structures independent of primary sequence bias and these offer new possibilities for the interpretation of conserved structural epitopes that are targeted by neutralizing antibodies and which are not explained from primary sequences alone [14].

Failure of the human Abs to neutralize the H7N7 avian strain is notable, since human H7N7 infections have been documented in 1959, 1977, 1981, 1996, and in the Netherlands in 2003 with 89 confirmed infections. Interestingly H7 subtypes have been found to have irregular ocular tropisms and in general humans are not considered to be at high infection risk, and $\mathrm{Ab}$ responses infrequently detected $[15,16]$. Hence between different avian strains, there appears to be a gradation of human antibodies for conserved inter-species protein epitopes and further study of other avian strains may confirm this.

There is need to validate our findings in models of in vivo infection and to determine whether there is an age-related antibody protection of $\mathrm{H} 5 \mathrm{~N} 1$ infection and pathogenicity, as has been suggested by Smallman-Raynor [13] and for which our unpublished preliminary findings are supportive. Also 


\section{Examination of Pooled Serum Abs for Binding to Human H1N1 \& H3N2 and Avian H5N1 Surface HA/NA Epitopes: Effect of H3N2 Adsorption}

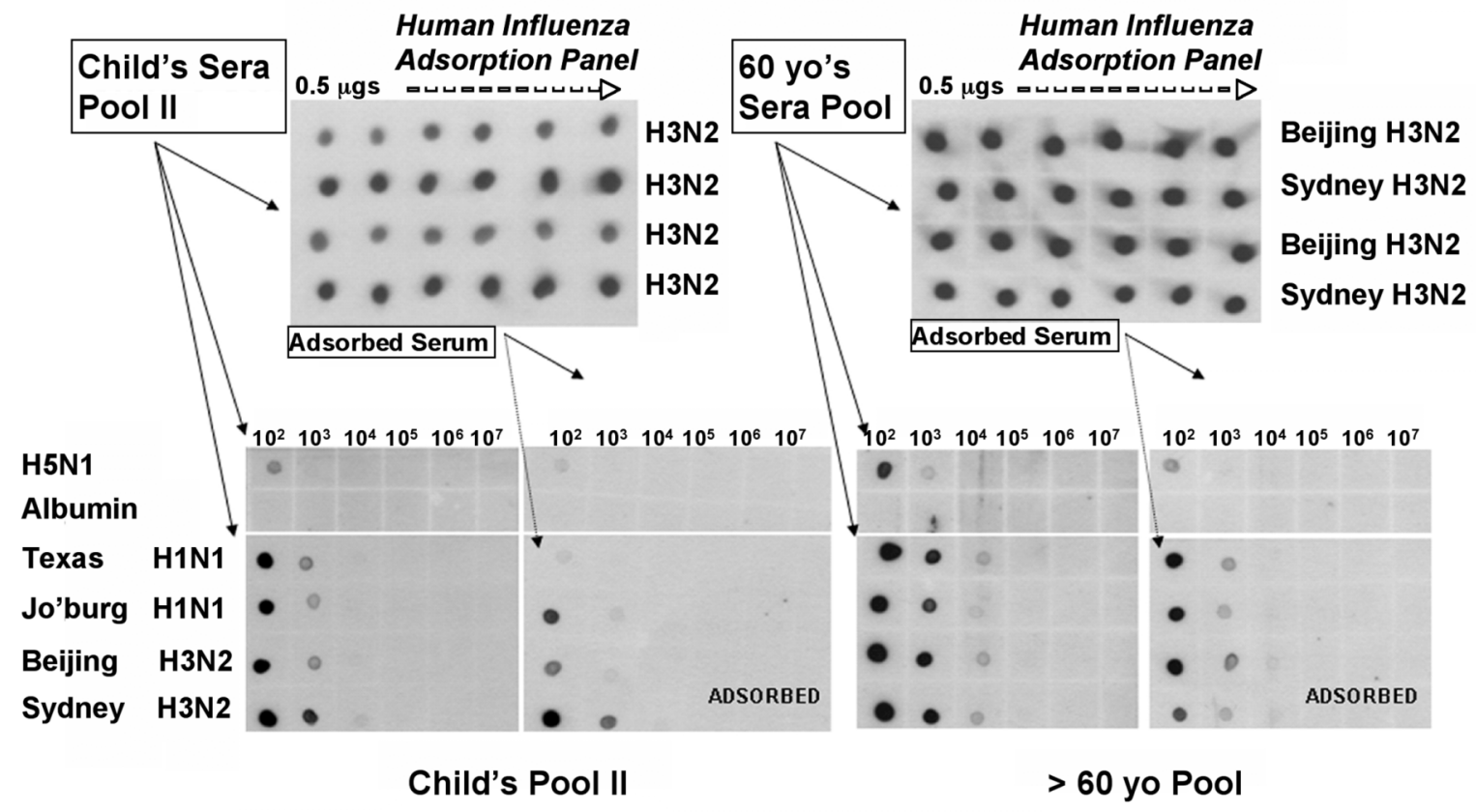

Fig. (5). Comparison of the binding responses of pooled adult or children's serum Abs for immobilized intact avian H5N1 and human H1N1 and H3N2 viruses: Proportional adsorption of H5N1 Ab binding by prior-exposure (adsorption) to human H3N2 viruses. As for the studies in Fig. (3) the binding of the pooled human antibodies from children (left hand panels) and adult sera (right hand panels) were examined in parallel either with or without prior exposure to H3N2 virus adsorption (top panels). For the latter, immobilized spot adsorption panels of $12 \times 0.5 \mathrm{ug}$ of the human H3N2 isolatesA/Beijing/32/1992 and A/Sydney/5/92 H3N2 were used. For detection and comparisons of the binding Abs with (i.e. adsorbed serum) or without exposure to the adsorption panel Ab binding for the child or adult pools were tested against serial dilutions of whole virus, equalized and matched for protein loads using ten fold dilutions of $500 \eta \mathrm{g}\left(\mathrm{i} . \mathrm{e}\right.$. $10^{2}-10^{7}$ ). Displayed are middle panels of intact whole H5N1 (chicken/Viet/8/2004) and albumin controls, and bottom panel tests of the two H1N1 and two H3N2 isolates. Shown is the data from a single passage (i.e., 2 hour incubation followed by washing) of the child or adult serum with the respective adsorption panels, with parallel treatment of the control serum pools as for those subjected to influenza adsorption. Note: the middle and bottom panels were processed separately because of the inter-strain competition for the anti-H5N1 binding Abs by unrelated human viruses, and to ensure optimal detection of the H5N1 binding activities free of in-test depletion. The bound human Abs were resolved by treatment with a 1/40,000 dilution of the anti-human HRP conjugate followed by chemiluminescence detection [7].

required and as highlighted in the recent Jordan report [1] is the need for resolution of the epitopes, pathways and mechanisms involved in the human antibody neutralization of H5N1 infections whether through targeting HA and NA [11] and possibly M2 [17]. It would be possible to do this by testing another virus containing a non-human neuraminidase gene, or to generate re-assortant viruses containing the avian $\mathrm{H} 5$ and a non-human neuraminidase. It is notable that with few exceptions (i.e. one human antibody study) to date all of the antibody epitopes of influenza have been identified from animal experiments. It is unknown whether there are conserved, cross-reactive and human-specific antibody epitopes for influenza.

Our on-going studies aim to profile those individuals with the highest titres so that preparations of enriched antiH5N1 Abs might be possible. From these candidates for quick response vaccine boosting may also be identified. A possibility bolstered by the report of enhanced anti H5 activities of healthy donors after human influenza vaccine boosting [12]. The protective value of using human neutral- izing anti-H5N1 Abs, generated from EBV treated B cells of a convalescent individual who had survived H5N1 infection, has also been shown in murine studies, along with evidence for $\mathrm{H} 5 \mathrm{~N} 1$ inter-clade cross-reactive antibody protection [10]. Of interest one of the monoclonal antibodies examined in the latter study despite being non- neutralizing in vitro gave significant protection from lethal injection in vivo [10]. Other studies have also shown that passive immune transfer of antibodies from $\mathrm{H} 5$ vaccine boosted mice were able to protect naive mice against homo-typic lethal challenge [18]. These findings and those from retrospective meta-analyses of convalescent blood product use in treating victims of the 1918 Spanish flu pandemic [19], indicate the potential value of passive anti-H5N1 Ab supplementation in the event of a H5N1 pandemic. While support for effective cross-reactive antibodies in nature can be gleaned from the findings of the Cleveland Family Study on the susceptibility to infection through the 1957 H2N2 pandemic, and suggests that preventative hetero-typic immunity may be accrued over the lifetimes of many individuals [20]. 


\section{ACKNOWLEDGEMENTS}

We wish to thank Dr. A. Hampson and the WHO laboratory, Parkville, Victoria, Australia, for the purified human influenza viruses. We are also grateful for the insight and helpful suggestions on the manuscript provided by Dr. Erica Wood, ARCBS, Victoria, Australia. The authors TD, NK and YW were recipients of University of Sydney, Faculty of Medicine Research Scholarships.

\section{REFERENCES}

[1] Subbarao K, Murphy, Fauci F, Anthony S. The Immunology of Influenza Infection: Implications for Vaccine Development. In: U.S. Dept of Health and Human Services NIH ed. The Jordan Report; Accelerated Development of Vaccines 2007: NIH, NIAID, 2007: 1-19.

[2] Palese P, Tumpey TM, Garcia-Sastre A. What can we learn from reconstructing the extinct 1918 pandemic influenza virus? Immunity 2006; 24(2): 121-4

[3] Taubenberger JK, Morens DM. 1918 Influenza: the mother of all pandemics. Emerg Infect Dis 2006; 12(1): 15-22.

[4] Cumulative Number of Confirmed Human Cases of Avian Influenza A/(H5N1) Reported to the World Health Organization (WHO) http: //www.who.int/csr/disease/avianVinfluenza/country/cases 2nd January 2008.

[5] Bui HH, Peters B, Assarsson E, Mbawuike I, Sette A. Ab and T cell epitopes of influenza A virus, knowledge and opportunities. Proc Natl Acad Sci USA 2007; 104(1): 246-51.

[6] Cottey R, Rowe CA, Bender, B. Current protocols in immunology: influenza virus. John Wiley and Sons, Inc., New York, NY, 2001.

[7] Sloane AJ, Raso V, Dimitrov DS, et al. Marked structural and functional heterogeneity in CXCR4: separation of HIV-1 and SDF1alpha responses. Immunol Cell Biol 2005; 83(2): 129-43.

[8] Lamb RA, Kug, RM. Orthomyxoviridae: The Viruses and their Replication. In: Fundamental Virology, Eds. Knipe DM and Howley PM 4 Edn: Lippincot Williams and Wilkins, 2001; pp. 725-769.
Qiu D, Tannock GA, Barry RD, Jackson DC. Western blot analysis of antibody responses to influenza virion proteins. Immunol Cell Biol 1992; 70(3): 181-91.

[10] Simmons CP, Bernasconi NL, Suguitan AL, et al. Prophylactic and therapeutic efficacy of human monoclonal antibodies against H5N1 influenza. PLoS Med 2007; 4(5): e178.

[11] Sandbulte MR, Jimenez GS, Boon AC, Smith LR, Treanor JJ, Webby RJ. Cross-reactive neuraminidase antibodies afford partial protection against $\mathrm{H} 5 \mathrm{~N} 1$ in mice and are present in unexposed humans. PLoS Med 2007; 4(2): e59.

[12] Gioia C, Castilletti C, Tempestilli M, et al. Cross-subtype immunity against avian influenza in persons recently vaccinated for influenza. Emerg Infect Dis 2007; 14(1) 121-128.

[13] Smallman-Raynor M, Cliff AD. Avian influenza A (H5N1) age distribution in humans. Emerg Infect Dis 2007; 13(3): 510-2.

[14] Fornasari MS, Parisi G, Echave J. Quaternary structure constraints on evolutionary sequence divergence. Mol Biol Evol 2007; 24(2): 349-51.

[15] Alexander DJ. Avian influenza viruses and human health. Dev Biol (Basel) 2006; 124: 77-84.

[16] Skowronski DM, Li Y, Tweed SA, et al. Protective measures and human antibody response during an avian influenza H7N3 outbreak in poultry in British Columbia, Canada. CMAJ 2007; 176(1): 4753.

[17] Tompkins SM, Zhao Z-S, Lo C-Y, et al. Matrix protein 2 vaccination and protection against influenza viruses, including subtype H5N1. Emerg Infect Dis 2007; 13(3): 426-34.

[18] Kong W-P, Hood C, Yang Z-Y, et al. Protective immunity to lethal challenge of the 1918 pandemic influenza virus by vaccination. Proc Natl Acad Sci USA 2006; 103(43): 15987-91.

[19] Luke TC, Kilbane EM, Jackson JL, Hoffman SL. Meta-analysis: convalescent blood products for Spanish influenza pneumonia: a future H5N1 treatment? Annals Inter Med 2006; 145(8): 599-609.

[20] Epstein SL. Prior H1N1 influenza infection and susceptibility of Cleveland Family Study during the H2N2 pandemic of 1957: an experiment of nature. J Infect Dis 2006; 193(11): 1613-4.

[21] Zhou Y-H, Chen Z, Purcell RH, Emerson SU. Positive reactions on Western Blots do not necessarily indicate the epitopes on antigens are continuous. Immunol Cell Biol 2007; 85: 73-8. 